\title{
OS EFEITOS DA REFLEXOLOGIA PODAL NO ENVELHECIMENTO
}

\section{ARTIGO DE REVISÃO}

DELAVAlentinA, Letícia Peruchi ${ }^{1}$, POLETTI, Sofia ${ }^{2}$

DELAVALENTINA, Letícia Peruchi. POLETTI, Sofia. Os efeitos da reflexologia podal no envelhecimento. Revista Científica Multidisciplinar Núcleo do Conhecimento. Ano. 06, Ed. 12, Vol. 07, pp. 50-65. Dezembro de 2021. ISSN: 24480959, Link de acesso: https://www.nucleodoconhecimento.com.br/saude/efeitos-dareflexologia, DOI: 10.32749/nucleodoconhecimento.com.br/saude/efeitos-dareflexologia

\section{RESUMO}

Introdução: O processo de envelhecimento biológico é inevitável a todo ser vivo. As transformações que o corpo do ser humano sofre são influenciadas por sua constituição genética no decorrer do tempo. O envelhecimento traz consigo muitas mudanças devido à idade, porém, fatores extrínsecos podem influenciar no envelhecimento biológico, acarretando doenças crônico-degenerativas, limitações funcionais e cognitivas, com quadros de ansiedade e depressão. Apesar do perfil idoso brasileiro, nos últimos anos, ter aumentado, o setor de saúde, ainda se encontra despreparado, mesmo com a implementação de políticas públicas de saúde, como: as Práticas Integrativas e Complementares (PICs), com diferentes métodos e técnicas de terapias naturais para complementar os tratamentos convencionais. Uma destas práticas é a Reflexologia Podal (RP), terapia realizada nos pés, onde pontos de reflexos estão diretamente ligados ao corpo todo. Estes, quando pressionados, influenciam reflexamente aos órgãos e glândulas, ocorrendo mudanças fisiológicas. Portanto, a presente revisão teve como questão norteadora: quais os efeitos da RP no envelhecimento? Objetivo: revisar na literatura os efeitos da RP no

\footnotetext{
${ }^{1}$ Graduanda do Curso de Bacharelado em Estética - FHO/Uniararas. ORCID: 9616944159163224.

${ }^{2}$ Orientadora. ORCID: 3126634954110201.
}

RC: 103522

Disponível em: https://www.nucleodoconhecimento.com.br/saude/efeitos-dareflexologia 
envelhecimento. Metodologia: Esta revisão foi aprovada pelo Comitê de Ética e Pesquisa da FHO, № 236/2020, com pesquisas nas bases de dados do Google Acadêmico, PubMed, SciELO, sem filtros, incluindo artigos clínicos nos idiomas português, inglês e espanhol. As palavras-chave utilizadas foram: reflexologia; envelhecimento; terapia, e as associações de palavras foram: envelhecimento e terapia nos pés; reflexologia em idosos, com as respectivas traduções em inglês e espanhol. Considerações Finais: A RP tem como principais benefícios o relaxamento, melhora a circulação sanguínea e influência na homeostasia das funções do corpo, amenizando estresse, pressão arterial, ansiedade, dores musculoesqueléticas, dentre outras. Assim, RP é considerada uma PIC, e pode ser uma grande aliada, não somente do processo de envelhecimento intrínseco, mas também um complemento para atenuação das comorbidades crônicas não-transmissíveis decorrentes do processo de envelhecimento extrínseco. Portanto, a RP possui efeitos positivos no envelhecimento.

Palavras-chave: reflexologia, envelhecimento, terapia.

\section{INTRODUÇÃO}

O envelhecimento intrínseco caracteriza-se como um processo fisiológico do nosso organismo, responsável por transformações influenciadas pela constituição genética ao decorrer do tempo. Dentro destas diferenças que o corpo apresenta, temos a diminuição e o embranquecimento dos cabelos, da espessura e da capacidade de sustentação da pele, redução na estatura da pessoa, aumento da amplitude do nariz e orelhas, do tecido adiposo e redução de tecidos muscular e ósseo (KUWAE et al., 2015).

Devido as diversas mudanças que o corpo do idoso apresenta ao longo dos anos, ocasiona-se então linhas de expressões, flacidez, rugas, bolsas orbitais, alteração na cavidade bucal, surgimento do arcus senilis (círculo branco em torno da córnea), modificação da língua, devido à grande perda de suas papilas gustativas e perda do paladar, desgastes nos dentes, desequilíbrio e diminuição em muitos casos de 
algumas funções motoras. A deposição do tecido adiposo geralmente ocorre ao redor das vísceras como rins, coração e no tronco (CORDEIRO et al., 2014).

Apesar do perfil do idoso brasileiro nos últimos anos ter aumentado, o setor de saúde ainda se encontra despreparado. Devido à idade se acarretam, na maioria dos casos, doenças crônico-degenerativas, ocasionando limitações funcionais e cognitivas. Com essa instabilidade na saúde, os idosos tendem a ficar mais próximos de seus familiares, e estes, muitas vezes, não estão preparados ou não aceitam este tipo de situação, ajudando a aumentar ainda mais a procura por Instituições de Longa Permanência para Idosos, e devido a isto, o grande número de casos de depressão nesta faixa etária (NÓBREGA et al., 2015).

Milhões de pessoas atualmente, independente da faixa etária, sofrem com quadros de ansiedade e depressão. Estes tendem a ser tratados primeiramente com acompanhamentos psicológicos e em alguns casos psiquiátricos, também sendo indicados aos pacientes alguns tipos de fármacos como ansiolíticos. Porém, muitos médicos estão utilizando a abordagem da Medicina Integrativa (MI), combinando assim a terapia convencional com a complementar (SCHIFF et al., 2020).

Este tipo de Medicina tem uma abordagem holística, sendo foco do tratamento os cuidados com bem-estar e saúde do indivíduo. Embora esta Medicina seja citada como uma que terá grandes resultados na vida do mesmo, pois seus potenciais são amplos diante da proposta de combinar diferentes tradições, fornecendo cuidado e a cura para o corpo, mente e espírito, e combinando a atuação de diferentes tipos de profissionais, ainda seu alcance integrativo para a área da saúde não é inteiramente identificado (KRACIK; PEREIRA; ISER, 2019).

Dentro das terapias holísticas, isto é, das Práticas Integrativas e Complementares (PICs), a Reflexologia Podal (RP) vem se destacando como método complementar para saúde. A RP parte de um princípio de que o corpo humano é dividido em diversos meridianos, estes responsáveis pela divisão das regiões que tem ligação direta com pontos reflexos encontrados principalmente nos pés, mas também podendo ser

RC: 103522

Disponível em: https://www.nucleodoconhecimento.com.br/saude/efeitos-dareflexologia 
encontrados nas mãos e orelhas. Estes pontos reflexos, que estão ligados diretamente em cada parte de nosso corpo e microssistemas, quando pressionados corretamente podem auxiliar na diminuição da dor, no relaxamento e na eliminação de toxinas, devolvendo ao corpo a homeostasia (SUS, 2020).

Os benéficos que são encontrados na RP são alívio de problemas digestivos, dores musculares, combate ao estresse, melhora da circulação sanguínea, grande estímulo da produção hormonal de endorfina, de receptores sensoriais e o sistema nervoso. Por esta técnica ser realizada nos pés e não ser invasiva, a mesma pode ser aplicada em qualquer ambiente, não trazendo desconforto na privacidade do paciente, nem necessitando de equipamentos especiais (DIAS et al., 2015).

Segundo Saraiva et al. (2015), durante o desenvolvimento das sessões de RP, observou-se não apenas a promoção de relaxamento, mas também que os idosos necessitavam de afeto e atenção. A institucionalização para muitos idosos gera isolamento, solidão e carência afetiva, podendo também culminar em quadros de estresse e ansiedade. Assim, visto os benefícios citados acima, a questão norteadora baseou-se em responder: quais os efeitos da RP no envelhecimento? Dessa maneira, o presente estudo teve como objetivo verificar na literatura os efeitos da RP no envelhecimento.

\section{DESENVOLVIMENTO}

Este trabalho teve aprovação do Comitê de Ética e Pesquisa da Fundação Hermínio

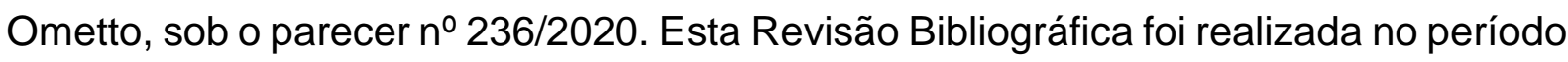
de fevereiro de 2020 a novembro de 2020, sendo utilizados artigos clínicos com idiomas inglês, espanhol e português, nas bases de dados do Google Acadêmico e PubMed (Biblioteca Nacional de Medicina dos Estados Unidos da América), sem filtros, com as palavras-chave: reflexologia; envelhecimento; terapia. As palavras em associações foram: envelhecimento e terapia por zonas; envelhecimento e terapia nos pés; reflexologia em idosos e terapia nos pés com idosos. 
O envelhecimento intrínseco, é um processo natural que ocorre com o decorrer da vida do ser humano, este, também está sujeito ao envelhecimento extrínseco, onde condiz com hábitos do dia a dia em que o homem está exposto e que pode acarretar muitas doenças crônicas não transmissíveis (DCNT), como o aumento da pressão arterial, diabetes, menopausa na mulher e andropausa no homem, doenças de tireoide, osteoartrite cervical, constipação, dentre outras (SILVA et al., 2015).

O processo de envelhecer é uma manifestação biológica do nosso corpo. Este, pode ser classificado em dois tipos, sendo o primeiro deles o intrínseco, mais conhecido como "natural", caracterizado por ser cronológico, ou seja, ocorre pelo avanço da idade. E o outro tipo de envelhecimento encontrado é o extrínseco, que está inteiramente ligado a fatores ambientais, que interferem a curto e longo prazo na vida do ser humano (geralmente acelera o processo normal), (Tabela 1) (SIMÕES; MOURA; MOREIRA, 2016)

Tabela 1: Causas do envelhecimento intrínseco e extrínseco.

\begin{tabular}{|l|lr}
\hline ENVELHECIMENTO & ENVELHECIMENTO \\
INTRÍNSECO & EXTRÍNSECO \\
\hline -Avanço da idade, & -Fatores ambientais \\
-Fatores genéticos & -Tabaco, Álcool \\
-Estado hormonal & -Radiação ultravioleta, \\
-Estresse oxidativo & -Poluição \\
& -Estilo de vida \\
& -Sedentarismo \\
& -Alta ingestão calórica. \\
\hline
\end{tabular}

Fonte: Elaborada pelo autor e adaptada de Alves; Esteves; Trelles, 2013.

Um dos fatores que compromete de forma considerável o envelhecimento intrínseco é o estresse oxidativo que atua no organismo de forma sistêmica, gerando uma cascata de eventos que podem desencadear diferentes DCNT interligadas, e que vai 
se tornando perigoso ao longo dos anos. Quando a produção de espécies reativas de oxigênio excede sua degradação acontece o estresse oxidativo (Figura 1) (LEITE et al., 2012).

Fig. 1. Representação da célula normal (A), célula atacada com radicais livres (B) e uma célula com estresse oxidativo $(\mathrm{C})$.

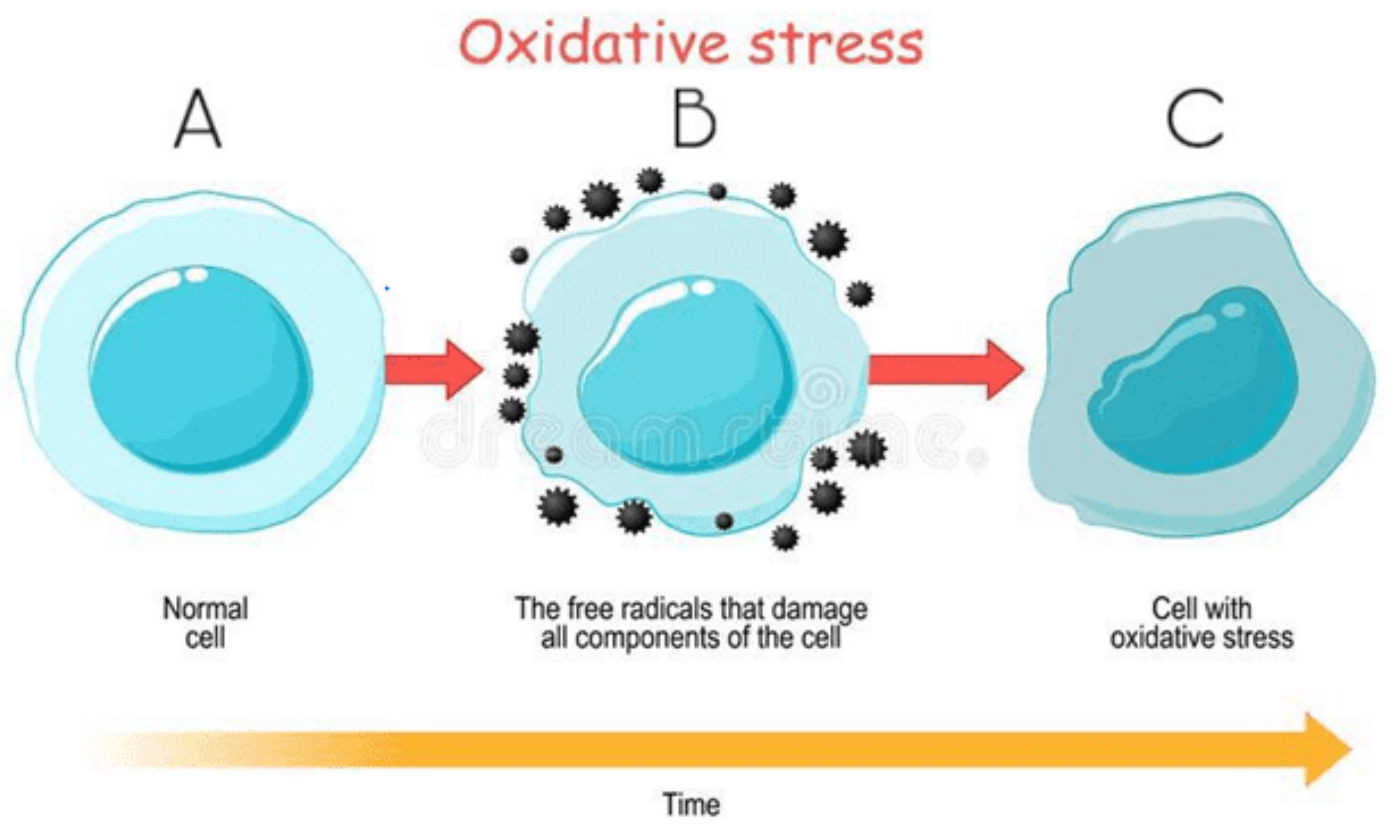

Fonte: $h$ ttps://pt.dreamstime.com/illustration/radicais.html

O estresse oxidativo é visto como um desequilíbrio entre a produção das espécies reativas de oxigênio (ERO), e doenças tem sido associada ao envelhecimento relacionadas à ação das espécies reativas de oxigênio (GOTTLIEB; MORASSUTTI; CRUZ, 2011). Continuamente o estresse oxidativo é gerado no organismo, e pode ser atenuado ou agravado mediante os hábitos da vida diária, como estilo de vida ativo, dieta, tabagismo consumo de álcool, exposição a radiações e a substâncias toxicas (LEITE et al., 2015).

O processo de envelhecimento pode ter influência na qualidade de sono, mas a menopausa aparentemente acentua esse processo gradual. No período em que a

RC: 103522

Disponível em: https://www.nucleodoconhecimento.com.br/saude/efeitos-dareflexologia 
mulher passa pela menopausa, ela tem grandes chances do aparecimento de oscilações no humor, acarretando a ansiedade, depressão e fragilidade emocional. Vários fatores podem estar também associados a estas alterações no humor, porém, a que tem a maior influência é a menopausa (TERAUCHI et al., 2014).

A maturação do sistema muscular completa no ser humano acontece entre os 20 e 30 anos de idade. Esses músculos variados que compõe este sistema, são formados por agrupamentos de fibras musculares distintas que aumentam gradativamente durante a evolução dos anos na vida da pessoa. Quando o ser humano atinge seus 30 anos de idade, ocorre uma diminuição da densidade muscular, devido a uma perda seletiva e gradual dessas fibras esqueléticas que são substituídas por colágeno e tecido adiposo (ESQUENAZI; SILVA; GUIMARÃES, 2014).

O envelhecimento visto biologicamente, é um processo progressivo e dinâmico, que ocorrem modificações da morfologia, funcionalidade e bioquímica, acarretando uma vulnerabilidade maior a patologias e uma diminuição da capacidade funcional. Esta fase da vida que é repleta de preconceitos, faz com que o idoso sofra muita dificuldade na inserção a sociedade, prejudicando ainda mais a vida do mesmo e acabando na maioria dos casos se isolando de todos (QUINTANA et al., 2014).

O aumento desta faixa etária no Brasil segundo a Pesquisa Nacional por Amostra de Domicílios Contínua - Características dos Moradores e Domicílios, do IBGE se manteve desde 2012 em constante crescimento, chegando no ano de 2017 a 30,2 milhões e tendo um aumentando de 4,8 milhões de habitantes idosos em território brasileiro no ano seguinte. A maioria desse grupo que faz parte do crescimento de $18 \%$ é representado pelo sexo feminino sendo, 16,9 milhões mulheres e 13,3 milhões de homens (IBGE, 2018).

Esse aumento na expectativa de vida necessita de uma grande atenção dos profissionais da saúde, da sociedade e do Estado, ou seja, um investimento maior na atenção primária, melhorando para que todos consigam ter acesso a mesma, para um 
envelhecimento digno, independentemente de ser uma população mais carente (CAMARGOS; GONZAGA, 2015).

O envelhecimento biológico, é considerado normal apesar de ter modificações nos sistemas de neurotransmissores e alterações microscópicas nos neurônios. O Sistema Nervoso Central apesar de não ser capaz de readquirir o neurônio, ele consegue diminuir o impacto dessas alterações, pois, no cérebro humano existem mais neurônios do que nosso corpo realmente precisa, este fato é chamado de redundância (SANTOS, 2014).

Como já foi observado em vários estudos, a capacidade de realização de tarefas autonomamente do nosso cotidiano, é reduzida conforme envelhecemos, esta diminuição é decorrente de várias alterações de todo nosso sistema biológico, órgãos e também tem grande influência dos fatores psicológicos e também sociais que ajudam ainda mais nesta degeneração funcional (CARVALHO, 2014).

Nosso Sistema Único de Saúde (SUS), atualmente oferece de forma gratuita vários procedimentos de PICS. Estas, veem sendo muito indicadas pelos profissionais específicos de acordo com as necessidades de cada caso cada dia mais. Há um crescente número de profissionais habilitados e capacitados e na valorização dessa prática, também possui grande número de evidências científicas que comprovam sua eficácia em conjunto a medicina convencional (SUS, 2020).

No Brasil este assunto vem tomando cada vez mais força devido a implementação no SUS, que zela pela busca da oferta de maneiras diferentes da prática do autocuidado e zelo com a saúde e o bem-estar mental, físico e emocional. Encontramos 29 tipos de PICS dentro do SUS, sendo Aromaterapia, Ayurveda, Biodança, Meditação, Quiropraxia, Reflexologia, dentre outras (MINISTÉRIO DA SAÚDE, 2006).

Considerada uma terapia alternativa e complementar, a Reflexologia acredita no princípio da existência de pontos reflexos nos pés e mãos, que estão diretamente ligados a cada glândula, órgão e estrutura do nosso corpo (Figura 1). Quando se 
estimula essas áreas reflexas, pode diminuir os níveis de tensões nervosas, auxiliando em uma gradativa recuperação do bem-estar (SOLÉ, 2019).

Fig. 1 - Representação das zonas reflexas da planta e lateral dos pés

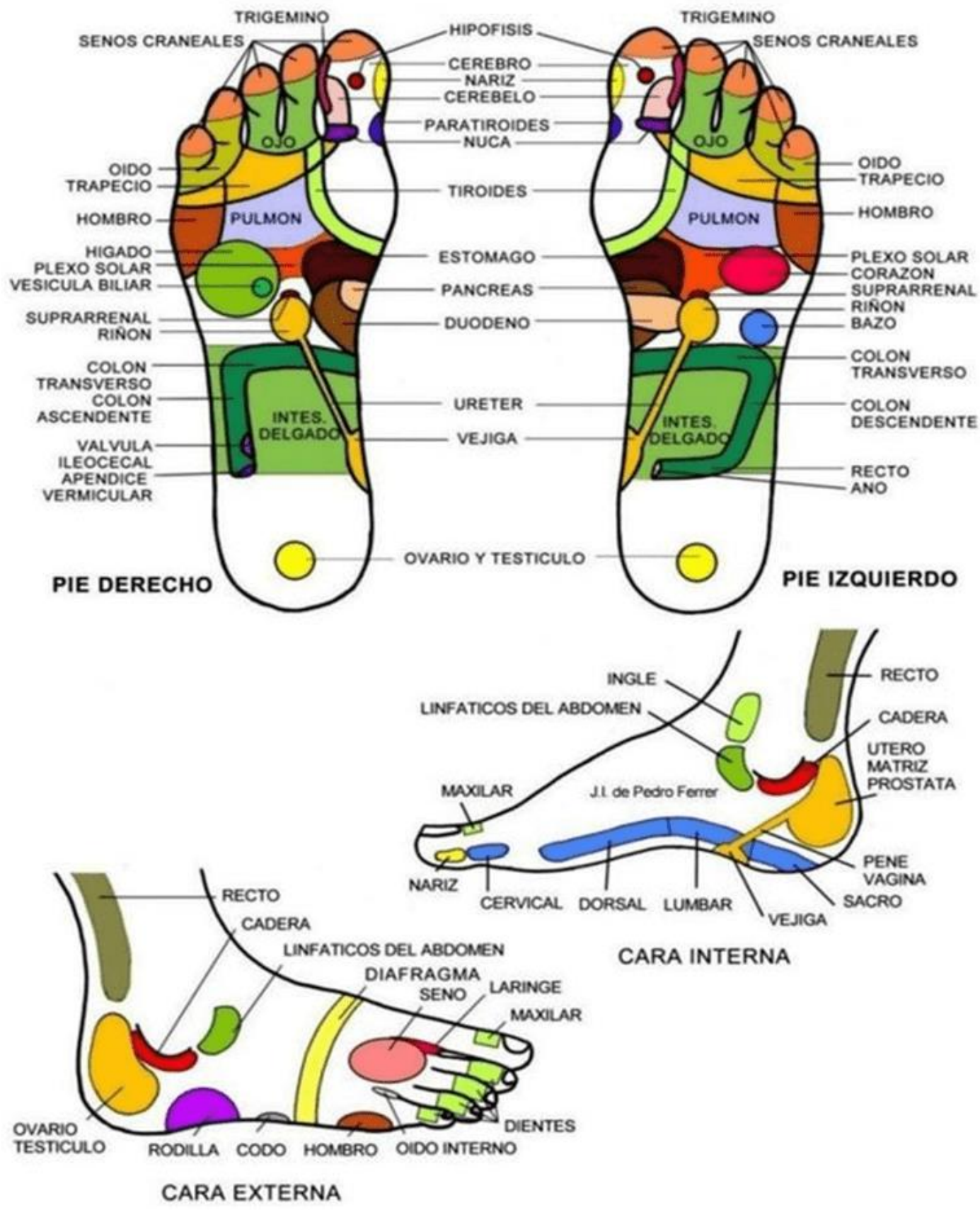

Fonte: https://br.pinterest.com/pin/771804454860273056/

RC: 103522

Disponível em: https://www.nucleodoconhecimento.com.br/saude/efeitos-dareflexologia 
Os estímulos nestas áreas reflexas nos pés, pode ser chamado de Reflexologia Podal (RP), com manipulação por meio dos dedos ou de objetos, a intensidade destes devem ser alternadamente ou contínuas e sempre suportável pelo paciente, por cerca de 20 segundos a 30 minutos. Os efeitos dessa técnica são comprovados em muitos estudos, que variam desde complementação em tratamentos de patologias musculoesqueléticas, de diminuição de pressão arterial, estresse e nervosismo, induzir ao sono, dentre outras enfermidades (SILVA et al., 2017).

Um dos principais benefícios encontrados na aplicação da RP é o relaxamento, o qual reduz a tensão, promovendo uma melhora na circulação sanguínea e linfática, e consequentemente levando a um melhor funcionamento do sistema nervoso central, auxiliando na homeostasia das funções do corpo. Assim, por meio da RP o paciente consegue prevenir o surgimento de doenças e manter a saúde (SANTO et al., 2016).

Segundo estudo de Saraiva et al. (2015), os idosos participantes da pesquisa residiam na Instituição de Longa Permanência para Idosos há mais de 5 anos e tinham acima de 70 anos, eles relataram no questionamento suas principais queixas, dentre elas estava, estresse, dor nos braços e pernas, coluna, insônia, pressão baixa, e o que utilizavam para melhorar, e o que implicava na saúde de cada um deles. Após o final da pesquisa a realização da RP nos idosos teve uma boa aceitação por eles, aliviando também um conforto gerado pela melhora das dores, ajudando no bem-estar.

Segundo Silva (2012), o estudo foi realizado em uma Instituição de Longa Permanência para Idosos, tendo como critério de inclusão residir nesta Instituição, estar orientado, consciente e aceitar participar da mesma. De 27 idosos, apenas 3 se encaixaram nesses critérios, sendo 2 mulheres e 1 homem com as idades de 70, 90 e 91 anos. Com os relatos dos idosos, pode-se observar notória diminuição nas dores físicas e melhora do bem-estar e condições de repouso e sono. Esta pesquisa possibilitou comprovar o uso da RP em idosos para cuidar não somente do corpo, mas da mente. 
Outro estudo, realizou uma intervenção terapêutica em pacientes com Osteoartrite Cervical (OC), atendidos pelo Serviço de Reabilitação Integral do Hospital Provincial Docente "Dr. Joaquín Castillo Duany "de Santiago em Cuba, desde julho de 2013 a dezembro de 2014. Foram selecionados 108 pacientes, distribuídos em 2 grupos de 54 integrantes cada, o Grupo Controle (GC) recebeu um tratamento convencional contendo, anti-inflamatórios, analgésicos e vitaminas. Já o Grupo Estudo (GE), foi utilizado a RP (30 minutos em dias alternados) até concluir 15 sessões. A idade é um dos principais fatores associados a aparição desta patologia, o GE foi o que mais obteve resultados, considerando assim a RP como um tratamento terapêutico para diminuição significativa dos sintomas em pacientes portadores de OC (VELÁZQUEZ et al., 2017).

O estudo de Dacal e Silva (2018), coletou dados de 59 pacientes, sendo 55 mulheres e 4 homens, com média de idade sendo 53,2 anos e um tempo médio de tratamento endócrino na unidade de 7,6 anos. Com relação as patologias encontradas nos pacientes, apresentavam obesidade, diabetes e doenças de tireoide. Das PICs oferecidas aproximadamente $70 \%$ deles foram atendidos com duas terapias, o Reike e a RP, $15 \%$ apenas com Reiki e outros $15 \%$ apenas RP. $69 \%$ deles (sendo a maioria, $\mathrm{N}=41)$ realizou até 5 sessões, $29 \%(\mathrm{~N}=17)$ realizaram entre 6 e 10 sessões e $2 \%(N=1)$ 15 sessões. Dentre as queixas deles estavam dores no corpo, ansiedade, estresse, cansaço, inchaço nas pernas e pés e ganho de peso; alguns pacientes também referiram pressão arterial alta, depressão, constipação, glicemia alta, sintomas de menopausa e cólicas. Observou-se nos resultados que não teve muita diferença entre o Reike e a RP quando utilizadas isoladamente, pacientes que realizaram até 5 sessões, obteve uma melhora de $62 \%$, o grupo que realizou de 6 a 10 sessões $69 \%$ de melhora e o que realizou acima de 15 sessões obteve $100 \%$ de melhora.

Em uma pesquisa realizada na casa dos avós da comunidade Habitacional do município de Manjibacoa, província de Las Tunas, Cuba, com 19 idosos, sendo 10 mulheres e 9 homens, na faixa etária de 50 a 70 anos; todos com queixas principais de hipertensão. Para a aplicação da RP foram intituladas algumas medidas sendo, não aplicar nas zonas lesionadas, lavar os pés antes e depois de cada sessão,

RC: 103522

Disponível em: https://www.nucleodoconhecimento.com.br/saude/efeitos-dareflexologia 
principais pontos a serem trabalhados foram os do sistema nervoso e sistema renal, realizado na posição deitada, com frequência de 5 dias por semana, com duração média de 20 minutos. Os valores no início da pesquisa variavam nos pacientes em 120-140 mmHg a sistólica e 80-95 mmHg a diastólica, já na primeira semana de sessões houve uma diminuição significativa, chegando aos valores de sistólica 110$130 \mathrm{mmHg}$ e diastólica $70-90 \mathrm{mmHg}$. A prática da RP teve uma influência positiva no tratamento da hipertensão em idosos, observando altos níveis de motivação e satisfação dentre os pacientes (RODRÍGUEZ et al., 2012).

Um ensaio clínico, randomizado, controlado e mascarado, teve como alvo pessoas com Diabetes Mellitus (DM) tipo 2, sendo 53 participantes com faixa etária de 18 a 65 anos, sendo randomizados em GC e GT. O GC foi integrado por 27 participantes com orientações de autocuidado com os pés (durante o rendimento do estudo, 5 pessoas desistiram de participar da pesquisa); já o GT, foi composto por 26 pessoas, que receberam orientações de autocuidado com os pés e participaram de 12 sessões de RP. Para conseguir avaliar os pacientes foi utilizado o Instrumento para avaliação da integridade tissular dos pés de pessoas portadoras de DM. Quando avaliado o comprometimento dos pés, tanto nos indicadores relacionados à pele e pelos como na sensibilidade, circulação sanguínea, e temperatura tissular, observou-se que, antes de iniciar o estudo, os participantes do GT e GC apresentaram escores de comprometimento similares, mas que após as rotinas implementadas aos mesmos, todos que receberam a RP tiveram melhores desenvolvimentos em alguns aspectos referentes à pele e pelos (SILVA et al., 2015).

Outro estudo com ensaio clínico randomizado, realizado em uma Instituição de Repouso Particular, selecionou 103 idosos, mas só puderam participar aqueles que foram capazes de falar e se orientar, que não utilizavam laxantes, não tinham Alzheimer ou demência, nenhum destes pacientes tiveram uma rotina de exercícios específicos e nem modificaram suas dietas. Durante o estudo um dos pacientes desenvolveu um quadro de emergência e teve que ser utilizado laxantes, excluindo assim o mesmo da pesquisa. Estes idosos foram divididos em dois grupos sendo o Grupo Experimental (GE), receberam RP com sentido horário para o cólon 3 vezes na RC: 103522

Disponível em: https://www.nucleodoconhecimento.com.br/saude/efeitos-dareflexologia 
semana por 30 minutos cada sessão, durante 1 mês, e o Grupo Controle (GC), foi aplicado uma massagem similar do GE, porém, sem pressão. Para avaliar estes pacientes foram utilizadas as seguintes regras, Formulário de solicitação de informações para idosos, constipação Instrumento de Gravidade (CSI) e Qualidade da Constipação de Escala de vida (CQLS). De acordo com os resultados do estudo, os idosos apresentaram uma melhora da constipação com a implementação da RP na rotina, tendo um aumento de $2,2 \%$ na taxa de defecação entre eles (INKAYA; TUZER, 2020).

\section{CONSIDERAÇÕES FINAIS}

São notórias as diferenças físicas e psicológicas em que o ser humano enfrenta ao decorrer da vida. Como foi visto ao longo do estudo cada dia mais a faixa etária idosa cresce em todo o mundo e frente a isso é de extrema importância a implementação e reconhecimento das PICs, onde busque formas de cuidados mais integralistas e humanizados.

De acordo com o presente estudo, pode-se concluir que, a RP é considerada uma PIC, e pode ser uma grande aliada, não somente do processo de envelhecimento intrínseco, mas também um complemento para atenuação das comorbidades crônicas não-transmissíveis decorrentes do processo de envelhecimento extrínseco. Portanto, respondendo a questão norteadora: quais os efeitos da RP no envelhecimento? Concluímos que a RP é uma terapia complementar que possui efeitos positivos no envelhecimento, promovendo o relaxamento, melhorando a circulação sanguínea, influenciando na homeostasia das funções do corpo, amenizando estresse, pressão arterial, ansiedade, dores musculoesqueléticas, dentre outras.

\section{REFERÊNCIAS}

ALVES, R.; ESTEVES, T. C.; TRELLES, M. A. Factores intrínsecos y extrínsecos implicados en el envejecimiento cutáneo. Cirugía Plástica Ibero-Latinoamericana, Espanha, v. 39, n. 1, P. 91-92, 2013. DOI: 10.4321/S0376-78922013000100013

RC: 103522

Disponível em: https://www.nucleodoconhecimento.com.br/saude/efeitos-dareflexologia 
BRASIL, Ministério da Saúde. Política nacional de práticas integrativas e complementares no SUS: atitude de ampliação de acesso. Brasília, 2006. Disponível em <https://bvsms.saude.gov.br/bvs/publicacoes/pnpic.pdf> Acesso em 03 março 2020.

CAMARGOS, M. C. S.; GONZAGA, M. R. Viver mais e melhor? Estimativas de expectativa de vida saudável para a população brasileira. Caderno de Saúde Pública, Rio de Janeiro, v. 31, n. 7, p. 1460-1472, 2015. DOI: 10.1590/0102$311 \times 00128914$.

CARVALHO, J. Pode exercício físico ser um bom medicamento para o envelhecimento saudável? Revista Acta Farmacêutica Portuguesa, Portugal, v. 3, n. 2, p. 123-130, 2014. Disponível em: < http://www.actafarmaceuticaportuguesa.com/index.php/afp/article/view/52>. Acesso em: 20 ago. 2020.

CORDEIRO, J. et al. Efeitos da atividade física na memória declarativa, capacidade funcional e qualidade de vida em idosos. Revista Brasileira de Geriatria e Gerontologia, Rio de Janeiro, v. 17, n. 3, p. 541-552, 2014. DOI: 10.1590/18099823.2014.13006

DIAS, D. M. et al. Efeitos da Reflexologia Podal sobre respostas cardiovasculares e sintomas específicos em idosos de um lar de longa permanência de Londrina - Estudo Piloto. X EPCC - Encontro Internacional de Produção Científica Londrina, 2015. Disponível em: <http://rdu.unicesumar.edu.br/handle/123456789/1921>. Acesso em: 20 abr. 2020.

DACAL, M. P. O.; SILVA, I, S. Impactos das práticas integrativas e complementares na saúde de pacientes crônicos. Revista Saúde e Debate, Rio de Janeiro, v. 42, n. 118, p. 724-735, 2018. DOI: 10.1590/0103-1104201811815.

RC: 103522

Disponível em: https://www.nucleodoconhecimento.com.br/saude/efeitos-dareflexologia 
ESQUENAZI, A.; SILVA, S. R. B.; GUIMARÃES, M. A. M. Aspectos fisiopatológicos do envelhecimento humano e quedas em idosos. Revista HUPE, Rio de Janeiro, v. 13, n. 2, p. 11-20, 2014. DOI: 10.12957/rhupe.2014.10124

GOTTLIEB M. G. V., MORASSUTTI A. L., CRUZ I. B. M. Transição epidemiológica, estresse oxidativo e doenças crônicas não transmissíveis sob uma perspectiva evolutiva. Scientia Medica, Porto Alegre, v. 21, n. 2, p. 69- 80, 2011.

IBGE - Instituto Brasileiro de Geografia e Estatística. Pesquisa Nacional por Amostra de Domicílios Contínua: características dos Moradores e Domicílios. 2018. Disponível em <https://agenciadenoticias.ibge.gov.br/agencia-noticias/2012agencia-de-noticias/noticias/20980-numero-de-idosos-cresce-18-em-5-anos-eultrapassa-30-milhoes-em-2017>. Acesso em 19 abril 2020.

INKAYA, B.; TUZER, H. Efecct of reflexology on the constipation status of elderly people. Journal Yonago Acta Medica, Japão, v. 63, n. 2, p. 115-121, 2020. DOI: 10.33160/yam.2020.05.007

KRACIK, M. L. A.; PEREIRA, P. M. B.; ISER, B. P. M. Medicina Integrativa: um parecer situacional a partir da percepção de médicos no Sul do Brasil. Revista Saúde em Debate, Rio de Janeiro, v. 43, n. 123, p 1095-1105, 2019. Disponível em: < https://www.scielo.br/pdf/sdeb/v43n123/0103-1104-sdeb-43-123-1095.pdf>. Acesso em: 15 abr. 2020.

KUWAE, C. A. et al. Concepção de alimentação saudável entre idosos na Universidade Aberta da Terceira Idade da UERJ: normas nutricionais, norma do corpo e normas do cotidiano. Revista Brasileira de Geriatria e Gerontologia, Rio de Janeiro, v. 18, n. 3, p. 621-629, 2015. DOI: 10.1590/1809-9823.2015.14224

LEITE, L. E. A. et al. Envelhecimento, estresse oxidativo e sarcopenia: uma abordagem sistêmica. Revista Brasileira de Geriatria e Gerontologia, v. 15, n. 2, p. 365-380, 2012. DOI: 10.1590/S1809-98232012000200018. 
LEITE L.M.G.S. et al. Estresse Oxidativo e Envelhecimento Humano: Uma Revisão Sistemática. Anais CIEH, v. 2, n. 1, 2015. DOI: 10.31415/bjns.v2i2.53

NÓBREGA, I. R. A. P. et al. Fatores associados à depressão em idosos institucionalizados: revisão integra. Revista Saúde e Debate, Rio de Janeiro, v. 39, n. 105, p.536-550, 2015. DOI: 10.1590/0103-110420151050002020

QUINTANA, J. M.; et al. A utilização da Classificação Internacional de Funcionalidade, Incapacidade e Saúde no cuidado aos idosos. Revista de Enfermagem Referência, Coimbra, v. 4, n. 1, p. 145-152, 2014. DOI: 10.12707/RIII12151.

RODRíGUEZ, C. P. et al. El massaje reflexológica podal en el tratamiento de adultos mayores hipertensos. Revista EFDeportes, Cuba, v. 1, n. 117, 2012. Disponível em: < https://www.efdeportes.com/efd117/masaje-reflexologico-podal.htm>. Acesso em: 15 abr. 2020.

SANTO, F. H. E. et al. Efectos de la reflexología de las manos en los ancianos hospitalizados. Revista Cultura de los cuidados, Valência, v. 20, n. 45, p. 147-153, 2016. DOI: 10.14198/cuid.2016.45.16

SANTOS, B. L. Efeitos do envelhecimento sobre o sistema nitrérgico dos núcleos da base em humanos. Tese de Doutorado da Universidade de São Paulo, Ribeirão Preto, p. 17-112, 2014. DOI: 10.11606/D.17.2014.tde-13052014-175226

SARAIVA, A. M. et al. Histórias de cuidados entre idosos institucionalizados: as práticas integrativas como possibilidades terapêuticas. Revista de Enfermagem da Universidade Federal de Santa Maria, Santa Maria, v. 5, n. 1, p. 131-140, 2015. DOI: $10.5902 / 2179769214211$

SCHIFF, E. et al. The effectiveness of foot reflexology in reducing anxiety and duration of labor in primiparas: An open-label randomized controlled trial. Complementary Therapies in Clinical Practice, Holanda, v. 38, n. 1, p. 1-9, 2020. DOI: 10.1016/j.ctcp.2019.101085

RC: 103522

Disponível em: https://www.nucleodoconhecimento.com.br/saude/efeitos-dareflexologia 
SILVA, A. I. S. et al. Reflexoterapia como técnica de relaxamento para profissionais em centro de terapia intensiva. Revista Ciência, Cuidado e Saúde, Belém, v. 16, v. 4, 2017. DOI: 10.4025/cienccuidsaude.v16i4.36334

SILVA, J. V. F. et al. A relação entre o envelhecimento populacional e as doenças crônicas não transmissíveis: sério desafio de saúde pública. Revista Ciências Biológicas e da Saúde, Maceió, v. 2, n. 3, p. 91-100, 2015. Disponível em: https://periodicos.set.edu.br/fitsbiosaude/article/view/2079/0>. Acesso em: 15 abr. 2020.

SILVA, N. C. M. et al. Reflexologia podal no comprometimento dos pés de pessoas com diabetes mellitus tipo 2: ensaio randomizado. Revista Latino-Americana de Enfermagem, Alfenas, v. 23, n. 4, p. 10-603, 2015. DOI: 10.1590/01041169.0036 .2594

SILVA, W. M. M. Reflexologia podal em idosos: promovendo o cuidado através dos pés. Universidade Federal de Campina Grande, p. 20-33, Cuité, 2012. Disponível em: < http://dspace.sti.ufcg.edu.br:8080/jspui/handle/riufcg/10883>. Acesso em 12 abr. 2020.

SIMÕES, R.; MOURA, M. M.; MOREIRA, W. W. Esperando a morte: o corpo do idoso institucionalizado. Revista Polêmica, Rio de Janeiro, v. 16, n. 3, p. 49-61, 2016. DOI: 10.12957/polemica.2016.25202

SUS. Sistema Único de Saúde. Práticas Integrativas e Complementares (PICS): quais são e para que servem. Disponível em: <https://www.saude.gov.br/saude-dea-z/praticas-integrativas-e-complementares>. Acesso em 3 mar 2020.

TERAUCHI, M. et al. Subgrouping of Japanese middle-aged women attending a menopause clinic using physical and psychological symptom profiles: a cross-sectional study. Journal BMC Women's Health, Tokyo, v. 14, n. 1, p. 148, 2014. Disponível em: < https://link.springer.com/article/10.1186/s12905-014-0148-z>. Acesso em: 15 ago. 2020.

RC: 103522

Disponível em: https://www.nucleodoconhecimento.com.br/saude/efeitos-dareflexologia 
VELÁZQUEZ, M. B. et al. Efectividad de la reflexologia podal em pacientes con artroses cervical. Revista Medisan, Santiago, v. 21, n. 2, p.144-150, 2017. Disponível em: < http://www.medisan.sld.cu/index.php/san/article/view/1333>. Acesso em: 20 set. 2020.

Enviado: Junho, 2021.

Aprovado: Dezembro, 2021. 